\title{
Some Optical Aspects of Thermally Evaporated Lead Oxide Thin Films
}

\author{
S.A. AlY*, M.A. KaID AND N.Z. El-SAYED \\ Physics Department, Faculty of Science, Minia University, Minia, Egypt \\ (Received October 15, 2012; in final form August 6, 2013)
}

The optical properties of lead oxide samples prepared using thermal evaporation technique on unheated glass substrates with different film thicknesses have been studied. The structural characteristics of a lead oxide sample was investigated using X-ray diffraction and it is confirmed to be in the amorphous state. The optical properties of the prepared films were studied by transmittance and reflectance measurements, and the integrated transmittance ( $T_{\mathrm{UV}}, T_{\mathrm{VIS}}$ and $\left.T_{\mathrm{Sol}}\right)$ in ultraviolet, visible and solar regions was calculated and found to be affected by film thickness. The dependence of absorption coefficient on wavelength was also reported. The energy gap was calculated and has been observed around $3.7 \mathrm{eV}$.

DOI: $10.12693 /$ APhysPolA.124.713

PACS: 77.55.-g, 81.15.-z, 78.20.-e, 78.20.Ci, 78.40.-q

\section{Introduction}

Transition metal oxides constitute a very interesting class of materials because of the various properties that they exhibit. Since heat transfer through windows is dominated by radiation, optical coatings can be used to improve thermal as well as visual comfort. In a warm climate this can be achieved by using solar control coatings, which decrease the transmittance of the non-visible near infrared radiation from the sun [1].

Many oxide materials are usually wide band gap compounds. The conductivity of oxides can vary from insulator to conductor, depending on the preparation conditions [2]. Due to their various optical and electronic properties, oxide-based compounds have been used for a wide variety of microelectronic and optoelectronic applications [3], such as electroluminescent device [4], magnetic memory [5], and dielectric layer [6].

In particular, the interaction between light and electrons in materials forms the basis for many interesting and practical effects. Those properties, such as photoconductivity and related phenomena, depend on materials composition, microstructure, energy band, carrier transport and others. Most of the reported optical and electronic properties of wide band gap oxides are based on single crystal or other bulk structures. Also, they appear to be helpful to understand the properties of oxide thin films in depth if the photoconductivity relaxation over a wide time range is known.

Lead oxide is of interest as a result of several of its properties. There are several phases of lead oxide which exhibit different levels of reflectance and thin films of lead oxide can be used as optical storage devices [7]. Lead oxide thin films also have applications as protective coatings for lead salt devices and gas sensors [8]. Lead oxide is also used as an adhesion promoter for thick films [9].

*corresponding author; e-mail: saaly61@hotmail.com

The preparation of lead oxide has received additional attention because lead oxide is a controlling factor in the quality of many lead-based ferroelectrics $[10,11]$. The preparation of these materials is often complicated by the high volatility of lead oxide at relatively low temperatures. Thin films of lead oxide have been produced in a low-temperature, low-pressure metal organic CVD process using $\mathrm{Pb}\left(\mathrm{C}_{2} \mathrm{H}_{5}\right)_{4}$ as a precursor [12]. Amorphous lead oxide was produced at room temperature by photochemical metal organic deposition [13].

This work aims to study the optical properties of some lead oxide thin films prepared using vacuum evaporation technique and correlate the dependence of the integrated transmittance features in the ultraviolet $\left(T_{\mathrm{UV}}\right)$, visible $\left(T_{\mathrm{Vis}}\right)$ and solar $\left(T_{\mathrm{Sol}}\right)$ ranges of the prepared samples relating to their film thickness.

\section{Experimental work}

\subsection{Sample preparation}

Lead oxide samples were prepared on unheated substrates by thermal evaporation using a high-purity lead oxide powder and a coating unit (type E306A Edwards Co.). The substrates were glass plates with rectangular shape, which were washed with alcohol, dried and then rubbed gently with cotton. The source to substrate distance was $16 \mathrm{~cm}$ apart. The heating filament was a conventional molybdenum boat. The pressure was brought down until a vacuum of about $4 \times 10^{-3} \mathrm{~Pa}$ was achieved.

\subsection{Sample characterization}

The film thickness was optically measured by multiple beam Fizeau fringes method at reflection using monochromatic light $\left(\mathrm{Hg}, \lambda_{\mathrm{g}}=546 \mathrm{~nm}\right)$.

To investigate the structure, a JEOL X-ray diffractometer (model JSDX-60PA) using nickel filtered $\mathrm{Cu} K_{\alpha}$ radiation $\left(\lambda_{K_{\alpha}}=1.5418 \AA\right)$ was employed to obtain diffraction patterns. The investigated sample is confirmed to be in the amorphous state as shown in Fig. 1. 


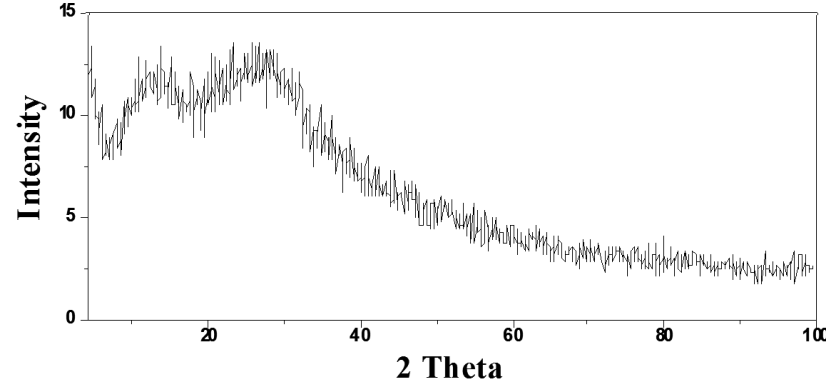

Fig. 1. X-ray diffraction pattern for a lead oxide sample of a film thickness of $200 \mathrm{~nm}$.

\subsection{Optical measurements}

The transmittance and reflectance measurements were carried out at normal incidence in the spectral range from 280 to $2400 \mathrm{~nm}$ using Shimadzu UV-3101PC:UV-VIS-NIR double-beam spectrophotometer, with a specular reflection attachment of $\mathrm{V}-\mathrm{N}$ type. The integrated ultraviolet, $T_{\mathrm{UV}}$, visible, $T_{\mathrm{Vis}}$, and solar, $T_{\mathrm{Sol}}$, transmittance of the prepared samples are derived by integrating, in the corresponding wavelength range of the measured transmittance, $T(\lambda)$, weighted by the solar spectrum $G(\lambda)$ at air mass 2 (AM2) [14], thus,

$$
T_{x}=\frac{\int_{\lambda 1}^{\lambda 2} T(\lambda) G(\lambda) \mathrm{d} \lambda}{\int_{\lambda 1}^{\lambda 2} G(\lambda) \mathrm{d} \lambda},
$$

where $x$ denotes $\mathrm{UV}$, visible or solar while $\lambda_{1}$ and $\lambda_{2}$ are the integration limits $280-380,380-780$, and $300-$ $2400 \mathrm{~nm}$, respectively. The absorption coefficient $\alpha$ was calculated from the following relation [15]:

$$
\alpha=\frac{1}{t} \ln \left(\frac{1-R(\lambda)}{T(\lambda)}\right),
$$

where $R(\lambda)$ and $T(\lambda)$ are, respectively, the spectral reflectance and transmittance at wavelength $\lambda$ and $t$ is the film thickness.

\section{Results and discussion}

3.1. Transmittance and reflectance measurements

Lead oxide thin films were examined in transmission using spectroscopy technique as shown in Fig. 2. An abrupt increase of transmittance with increasing wavelength up to about $400 \mathrm{~nm}$ was observed followed by a slight increase up to $2400 \mathrm{~nm}$.

Also, a strong dependence of transmittance on film thickness can be easily observed over the used wavelength range. Sample of high film thickness possessess a poor transmittance. It can be, also, observed that the transmittance in the NIR region for the sample of $82 \mathrm{~nm}$ thickness is quite high. Films used to overcoat a metal, must exhibit high infrared transmittance in order to preserve the infrared reflectance of the metal [16].

The dependence measured reflectance on wavelength is depicted in Fig. 3. A small variation in the average value of the spectral reflectance is noticed from about 20 to $35 \%$.

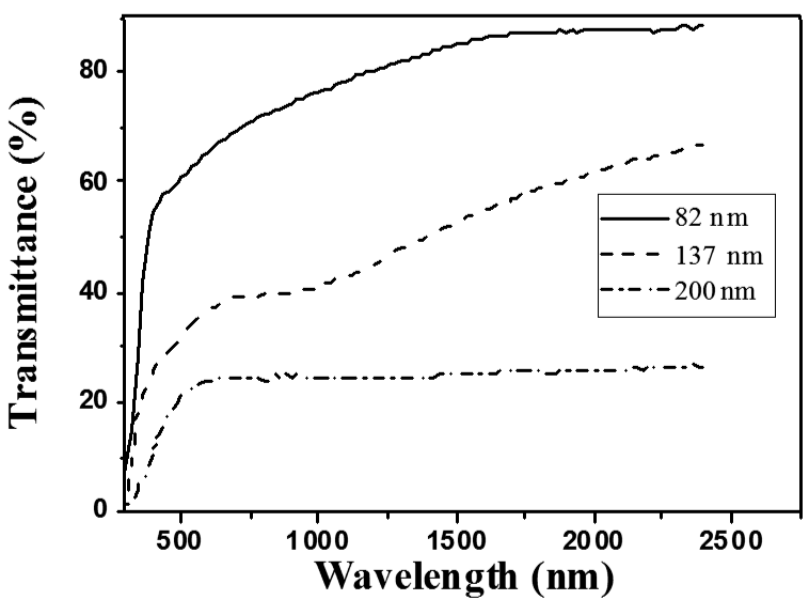

Fig. 2. Variation of spectral transmittance with wavelength for some lead oxide samples of different film thicknesses.

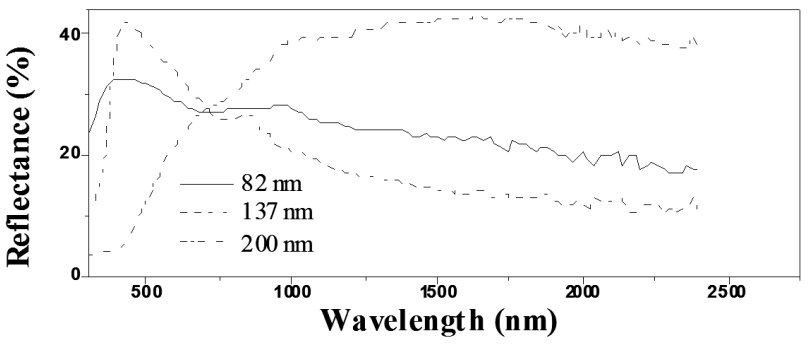

Fig. 3. Variation of spectral reflectance with wavelength for some lead oxide samples of different film thicknesses.

\subsection{Integrated transmittance}

The dependence of integrated transmittance $\left(T_{\mathrm{V} \text { is }}\right.$ and $\left.T_{\text {Sol }}\right)$ on film thickness is illustrated in Fig. 4. The dependence indicated qualitatively the same behaviour but there are some differences in the measured values. A considerable decrease was observed in both of $T_{\mathrm{Vis}}$ and $T_{\mathrm{Sol}}$ from 0.63 and 0.66 , respectively, to about 0.2 as the film thickness increases from $82 \mathrm{~nm}$ to $200 \mathrm{~nm}$.

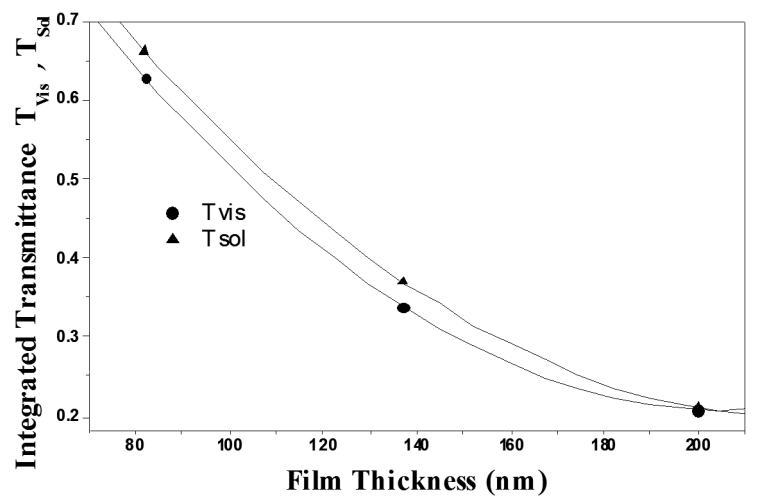

Fig. 4. The integrated transmittance $\left(T_{\mathrm{Vis}}\right.$ and $\left.T_{\mathrm{Sol}}\right)$ as a function of film thickness. 
Variation of $T_{\mathrm{UV}}$ on film thickness is depicted in Fig. 5 . The same dependence on film thickness as well as in case of $T_{\mathrm{Vis}}$ and $T_{\mathrm{Sol}}$ was observed except that the values of $T_{\mathrm{UV}}$ are quite low $\left(T_{\mathrm{UV}} \leq 0.22\right)$. Sample of $200 \mathrm{~nm}$ thickness exhibits a very low value $\left(T_{\mathrm{UV}}=0.03\right)$.

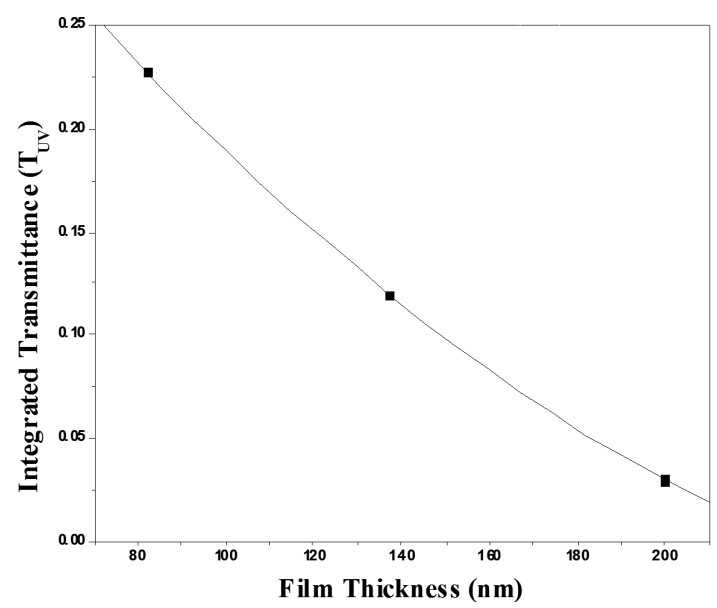

Fig. 5. The integrated transmittance $\left(T_{\mathrm{UV}}\right)$ as a function of film thickness.

The most harmful radiation in sunlight is the ultraviolet (UV) rays, which are the most energetic and thus most likely to break chemical bonds, leading to fading and degradation. Many organic materials, such as carpet, fabrics, paper, artwork, paints and wood can fade during the exposure to sunlight. For this reason, window selection can influence the type and intensity of transmitted radiation. Glass blocks all UV radiation below $300 \mathrm{~nm}$, but transmits UV from 300-380 nm [17]. Glass coated lead oxide with a film thickness around $80 \mathrm{~nm}$ can pass about $66 \%$ from the solar spectrum while transmit only about $22 \%$ from the UV rays. The thicker sample of $200 \mathrm{~nm}$ transmit only 0.03 from the UV rays. So, thermally evaporated lead oxide thin films can be predicted to be used as a UV-protective.

\subsection{Absorption coefficient and energy gap}

The absorption coefficient $\alpha$ has been calculated using Eq. (2) and its dependence on wavelength at two different samples has been investigated as shown in Fig. 6 . An abrupt decrease in the absorption coefficient value with increasing wavelength up to about $330 \mathrm{~nm}$, then no appreciable change was observed. The strong absorption and dispersion in the wavelength range indicates that the energy gap is located in that region (around $330 \mathrm{~nm}$ ).

The optical energy gap $\left(E_{\mathrm{g}}\right)$ of the films was estimated from the optical measurements. The absorption coefficient was found to follow the relation

$$
\alpha=\frac{A\left(h \nu-E_{\mathrm{g}}\right)^{1 / 2}}{h \nu}
$$

where $A$ is a constant.

The plot of $(\alpha h \nu)^{2}$ versus the photon energy in the absorption region for the samples of 82 and $137 \mathrm{~nm}$ thicknesses indicates direct allowed transition. The energy gap

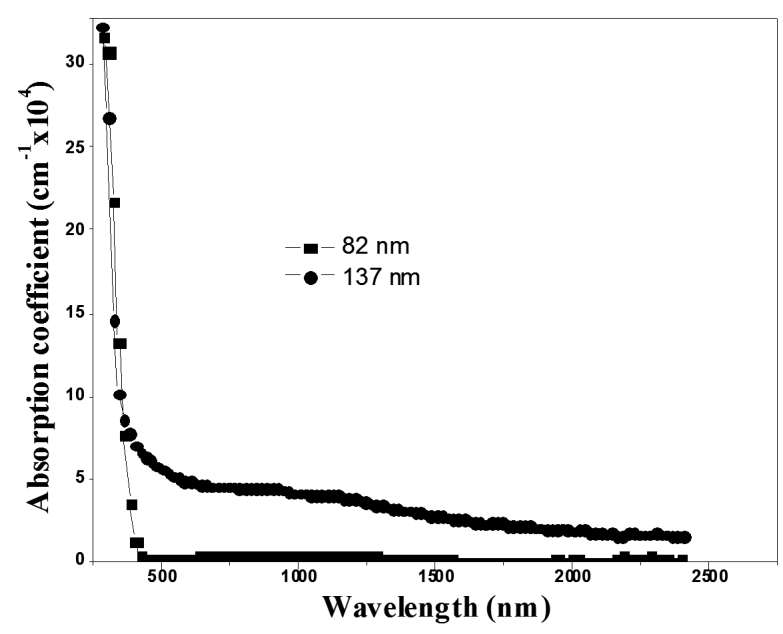

Fig. 6. Variation of absorption coefficient $\alpha$ with wavelength for two lead oxide samples of different film thicknesses.

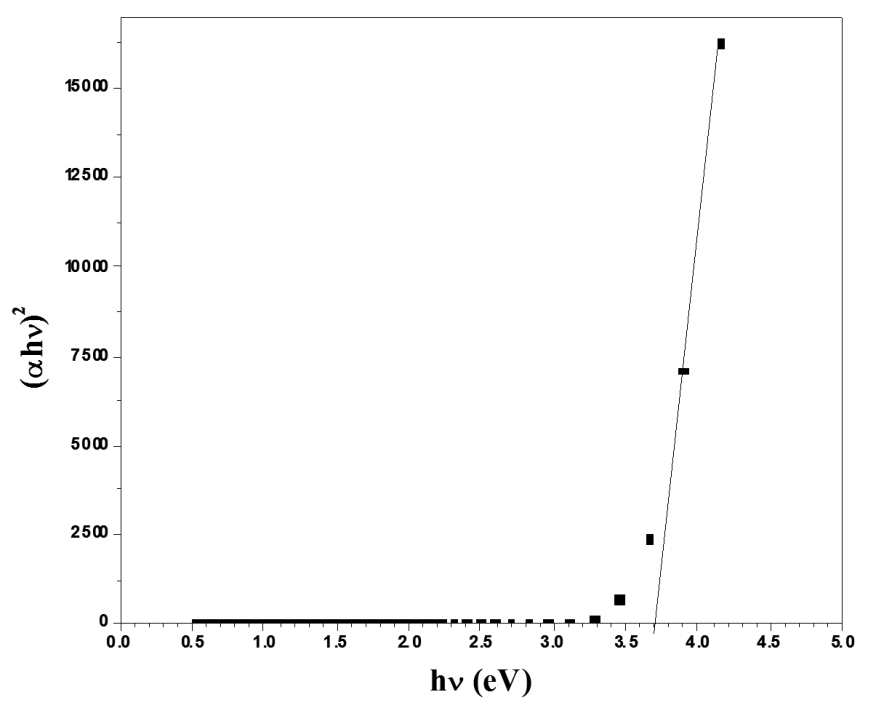

Fig. 7. $(\alpha h \nu)^{2}$ as a function of the photon energy $(h \nu)$ for a lead oxide sample of $82 \mathrm{~nm}$.

can be determined from the extrapolation of the linear portion with the photon energy axis and is illustrated in Figs. 7 and 8. It is noticed that the value of $E_{\mathrm{g}}$ is around $3.7 \mathrm{eV}$ for the two samples.

Unfortunately there is not much literature data available for the band gap in lead oxides. $E_{\mathrm{g}}$ values of 1.9 and $2.5 \mathrm{eV}$, respectively, were found for the tetragonal and orthorhombic structure of $\mathrm{PbO}$ [18]. For $\mathrm{Pb}_{3} \mathrm{O}_{4}$ an experimental value of $2.1-2.2 \mathrm{eV}$ is reported, while for $\mathrm{PbO}_{2}$ a value of $4.2 \mathrm{eV}$ has been measured for the $\alpha-\mathrm{PbO}_{2}$ phase and a value of $1.5 \mathrm{eV}$ has been reported for $\beta-\mathrm{PbO}_{2}$ $[19,20]$. 


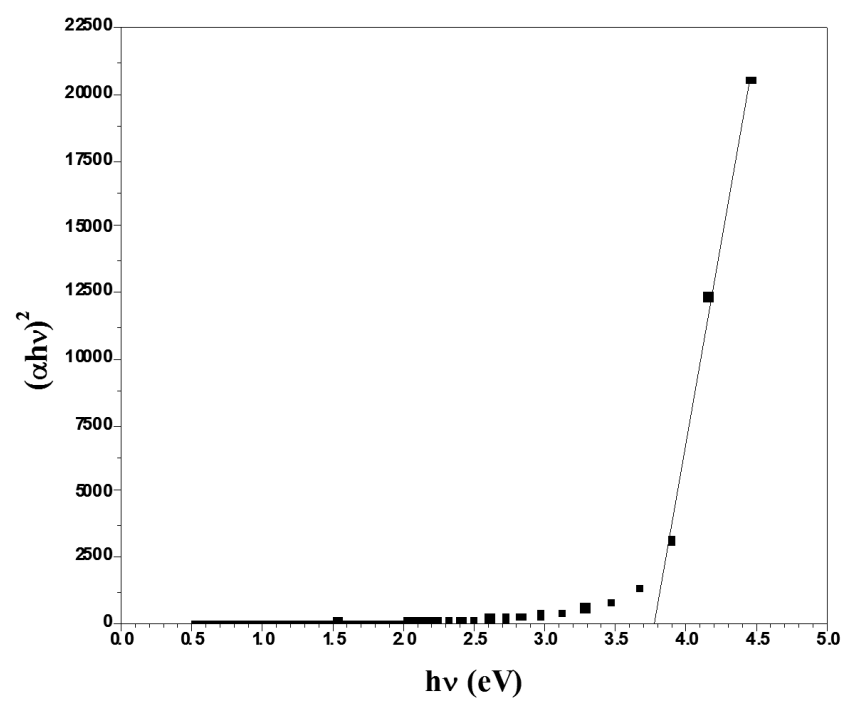

Fig. 8. $(\alpha h \nu)^{2}$ as a function of the photon energy $(h \nu)$ for a lead oxide sample of $137 \mathrm{~nm}$.

\section{Conclusion}

Lead oxide thin films have been prepared using thermal evaporation technique at room temperature on unheated glass substrate.

The optical investigations based on the spectrophotometric characteristics have confirmed that a considerable increase of transmittance with increasing wavelength up to about $400 \mathrm{~nm}$ is observed followed by a slight increase up to $2400 \mathrm{~nm}$. Also, sample of small film thickness possessess a high transmittance value. A small variation in the average value of the spectral reflectance is noticed from 20 to $35 \%$.

A considerable decrease was observed in both $T_{\mathrm{Vis}}$ and $T_{\text {Sol }}$ to about 0.2 as the film thickness increases. The same behaviour is also observed in case of $T_{\mathrm{UV}}$, except that its values are quite low $\left(T_{\mathrm{UV}} \leq 0.22\right)$. Thicker sample of $200 \mathrm{~nm}$ thickness exhibits a very low UV transmittance value $\left(T_{\mathrm{UV}}=0.03\right)$

A considerable decrease in the absorption coefficient value with increasing wavelength up to about $330 \mathrm{~nm}$ was observed as a result of transition across the semiconductor band gap in lead oxide. For wavelengths $>330 \mathrm{~nm}$ no appreciable change was observed.

The energy gap for two samples of different film thicknesses (82 and $137 \mathrm{~nm}$ ) is calculated and its value is located around $3.7 \mathrm{eV}$.

\section{References}

[1] L. Kullman, Component Sol of Smart Windows, Acta Universitatis Upsaliensis, Uppsala 1999.

[2] M. Fleischer, H. Meixner, J. Mater. Sci. Lett. 11, 1728 (1992).

[3] M. Passlack, E.F. Schubert, W.S. Hobson, M. Hong, N. Moriya, S.N.G. Chu, K. Konstadinidis, J.P. Mannaerts, M.L. Schnoes, G.J. Zydzik, J. Appl. Phys. 77, 686 (1995).

[4] J.H. Hao, Z. Lou, I. Renaud, M. Cocivera, Thin Solid Films 467, 182 (2004).

[5] E. Aubay, D. Gourier, Phys. Rev. B 47, 15023 (1993).

[6] J.H. Hao, W. Si, X.X. Xi, R. Guo, A.S. Bhalla, L.E. Cross, Appl. Phys. Lett. 76, 3100 (2000).

[7] S. Chao, Y.F. Fuang, Y.C. Chen, L. Yan, J. Phys. D 23, 955 (1990).

[8] L.D. Madsen, L. Weaver, J. Am. Ceram. Soc. 81, 988 (1998).

[9] M. Bersani, B. Morten, M. Prudenziati, J. Mater. Res. 12, 501 (1997).

[10] E. Hong, J.C. Shin, J. Choi, C.S. Wang, H.J. Kim, J. Mater. Res. 15, 1284 (2000).

[11] J.H. Kim, Y. Kim, A.T. Chien, F.F. Lange, J. Mater. Res. 16, 1739 (2001).

[12] R.S. Boorse, J.M. Burlitch, Chem. Mater. 6, 1509 (1994).

[13] S.L. Blair, C.W. Chu, R. Dammel, R.H. Hill, Proc. SPIE 3049, 829 (1997).

[14] C.G. Granqvist, C.M. Lampert, Science and Technology of Electrochromics, Optical Materials Technology for Energy Efficiency and Solar Energy, Toulouse 1992.

[15] F. Demichelis, G. Kaniadakis, A. Tagliferro, E. Tresso, J. Appl. Opt. 26, 1737 (1987).

[16] C.M. Lampert, C.G. Granqvist, Large Area Chromogenics: Materials and Devices for Transmittance Control, SPIE, Bellingham 1990.

[17] The Efficient Windows Collaborative Benefits.htm.

[18] S. Radhakrishnan, M.N. Kamalasanan, P.C. Mahendru, J. Mater. Sci. 18, 1912 (1983).

[19] T. Arai, J. Phys. Soc. Jpn. 15, 916 (1960).

[20] S. Venkatara, J. Geurts, H. Weis, O. Kappertz, W.K. Njoroge, R. Jayavel, M. Wuttiga, J. Vac. Sci. Technol. A 19, 2870 (2001). 